\title{
Statistical Error In Particle Simulations Of Low Mach Number Flows
}

N. G Hadjiconstantinou, A. L Garcia

This article was submitted to

First MIT Conference on Computational Fluid and Solid Mechanics Cambridge, MA

June $12-14,2000$

November 13, 2000

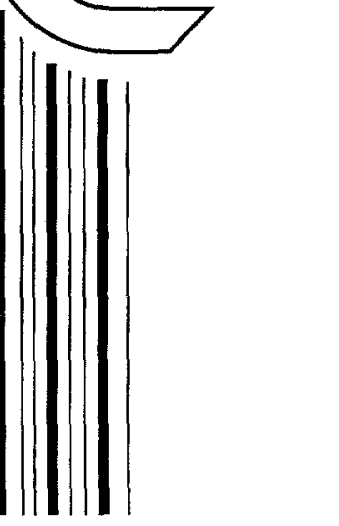




\section{DISCLAIMER}

This document was prepared as an account of work sponsored by an agency of the United States Government. Neither the United States Government nor the University of California nor any of their employees, makes any warranty, express or implied, or assumes any legal liability or responsibility for the accuracy, completeness, or usefulness of any information, apparatus, product, or process disclosed, or represents that its use would not infringe privately owned rights. Reference herein to any specific commercial product, process, or service by trade name, trademark, manufacturer, or otherwise, does not necessarily constitute or imply its endorsement, recommendation, or favoring by the United States Government or the University of California. The views and opinions of authors expressed herein do not necessarily state or reflect those of the United States Government or the University of California, and shall not be used for advertising or product endorsement purposes.

This is a preprint of a paper intended for publication in a journal or proceedings. Since changes may be made before publication, this preprint is made available with the understanding that it will not be cited or reproduced without the permission of the author.

This report has been reproduced

directly from the best available copy.

Available to DOE and DOE contractors from the

Office of Scientific and Technical Information

P.O. Box 62, Oak Ridge, TN 37831

Prices available from (423) 576-8401

http://apollo.osti.gov/bridge/

Available to the public from the

National Technical Information Service

U.S. Department of Commerce

5285 Port Royal Rd.,

Springfield, VA 22161

http://www.ntis.gov/

OR

Lawrence Livermore National Laboratory

Technical Information Department's Digital Library

http://www.1lnl.gov/tid/Library.html 


\title{
Statistical Error in Particle Simulations of Low Mach Number Flows
}

\author{
Nicolas G. Hadjiconstantinou \\ Mechanical Engineering Department \\ Massachusetts Institute of Technology \\ Cambridge, MA 02139 \\ and \\ Alejandro L. Garcia* \\ Center for Applied Scientific Computing \\ Lawrence Livermore National Laboratory \\ Livermore, $C A 94551$
}

Proceedings of the First MIT Conference on

Computational Fluid and Solid Mechanics, Elsevier, June 2001

\begin{abstract}
We present predictions for the statistical error due to finite sampling in the presence of thermal fluctuations in molecular simulation algorithms. The expressions are derived using equilibrium statistical mechanics. The results show that the number of samples needed to adequately resolve the flowfield scales as the inverse square of the Mach number. Agreement of the theory with direct Monte Carlo simulations shows that the use of equilibrium theory is justified.
\end{abstract}

\section{Introduction}

Recently much attention has been focused on the simulation of hydrodynamic problems at small scales using molecular simulation methods such as Molecular Dynamics (MD) [1] or the direct simulation Monte Carlo (DSMC) [2, 3]. Molecular Dynamics is generally used to simulate liquids while DSMC is a very efficient algorithm for simulating dilute gases. In molecular simulation methods the connection to macroscopic observable fields, such as velocity and temperature, is achieved through averaging appropriate microscopic properties over small regions of space. The simulation results are therefore inherently statistical and statistical errors due to finite sampling need to be fully quantified.

*Permanent address: Dept. Physies, San Jose State Univ., San Jose, CA 95192-0106 
Here we derive expressions for the magnitude of statistical errors due to thermal fluctuations in such simulations for the typical observables of interest, namely velocity, density and temperature. Although we shall make use of expressions from equilibrium statistical mechanics, the non-equilibrium modifications to these results are very small, even under extreme conditions [4].

\section{Statistical error due to thermal fluctuations}

We first consider the fluid velocity. In a particle simulation, the flow field is obtained by measuring the instantaneous center of mass velocity, $u$, for particles in a statistical cell volume. For steady flows, the statistical mean value of the local fluid velocity, $\langle u\rangle$, is estimated over $M$ independent samples. The average fluid velocity, $u_{0}$, is defined such that $\langle u\rangle \rightarrow u_{0}$ as $M \rightarrow \infty$. Let $N_{0}$ be the average number of particles in the statistical cell and $\delta u \equiv u-u_{0}$ the instantaneous fluctuation of the fluid velocity. From equilibrium statistical mechanics [5],

$$
\left\langle\delta u^{2}\right\rangle=\frac{k T_{0}}{m N_{0}}=\frac{c^{2}}{\gamma \mathrm{Ac} c^{2} N_{0}}
$$

where $T_{0}$ is the average temperature, $m$ is the particle mass, $k$ is Boltzmann's constant, $c$ is the sound speed, $\gamma=c_{P} / c_{V}$ is the ratio of the specific heats. The acoustic number $\mathrm{Ac}=c / c^{i}$ is ratio of the fluid's sound speed to the sound speed of a "reference" ideal gas at the same temperature $\left(c^{i}=\sqrt{\gamma k T / m}\right)$. This reference ideal gas has a ratio of specific heats $\left(\gamma^{i}\right)$ equal to the original fluid specific heat ratio, that is $\gamma^{i}=\gamma$.

We may define a "signal-to-noise" ratio as the average fluid velocity over its standard deviation; from the above,

$$
\frac{u_{0}}{\sqrt{\left\langle\delta u^{2}\right\rangle}}=\operatorname{AcMa} \sqrt{\gamma N_{0}}
$$

where $\mathrm{Ma}=u_{0} / \mathrm{c}$ is the local Mach number. This result shows that for fixed Mach number, in a dilute gas simulation $(\mathrm{Ac}=1)$ the statistical error due to thermal fluctuations cannot be ameliorated by reducing the temperature. However, when the Mach number is small enough for compressibility effects to be negligible, favorable relative statistical errors may be obtained by performing simulations at an increased Mach number (to a level where compressibility effects are still negligible).

The one-standard-deviation error bar for the sample estimate $\langle u\rangle$ is $\sigma_{u}=$ $\sqrt{\left\langle\delta u^{2}\right\rangle} / \sqrt{M}$ and the fractional error in the estimate of the fluid velocity is

$$
E_{u}=\frac{\sigma_{u}}{u_{0}}=\frac{1}{\sqrt{M N_{0}}} \frac{1}{\operatorname{AcMa} \sqrt{\gamma}}
$$

yielding

$$
M=\frac{1}{\gamma \mathrm{Ac}^{2} N_{0} \mathrm{Ma}^{2} E_{u}^{2}} .
$$


For example, with $N_{0}=100$ particles in a statistical cell, if a one percent fractional error is desired in a $\mathrm{Ma}=1$ flow, about $M=100$ independent statistical samples are required (assuming $\mathrm{Ac} \approx 1$ ). However, for a $\mathrm{Ma}=10^{-2}$ flow, about $10^{6}$ independent samples are needed. Since most particle methods require $10-100$ time steps between independent samples, this makes the resolution of the flow velocity computationally expensive for low Mach number flows.

Next we turn our attention to the density. From equilibrium statistical mechanics, the fluctuation in the number of particles in a cell is

$$
\left\langle\delta N^{2}\right\rangle=-N^{2} \frac{k T}{V^{2}}\left(\frac{\partial V}{\partial P}\right)_{T}=\kappa_{T} N_{0}^{2} \frac{k T_{0}}{V}
$$

where $\kappa_{T} \equiv-V^{-1}(\partial V / \partial P)_{T}$ is the isothermal compressibility. Note that for a dilute gas $\kappa_{T}=1 / P$ so $\left\langle\delta N^{2}\right\rangle=N$ and, in fact, the probability distribution of $N$ is Poissonian. The fractional error in the estimate of the density is

$$
E_{\rho}=\frac{\sigma_{\rho}}{\rho_{0}}=\frac{\sigma_{N}}{N_{0}}=\frac{\sqrt{\left\langle\delta N^{2}\right\rangle}}{N_{0} \sqrt{M}}=\frac{\sqrt{\kappa_{T} k T_{0}}}{\sqrt{M V}}
$$

where $V$ is the volume of the cell. The above expression can also be written as

$$
E_{\rho}=\frac{\sigma_{\rho}}{\rho_{0}}=\frac{\sqrt{\kappa_{T} / \kappa_{T}^{i}}}{\sqrt{M N_{0}}}
$$

where $\kappa_{T}^{i}=V / N_{0} k T_{0}$ is the isothermal compressibility of the reference dilute gas $\left(\gamma^{i}=\gamma\right)$ at the same density and temperature. Since $c \propto 1 / \sqrt{\kappa_{T}}$,

$$
E_{\rho}=\frac{1}{\sqrt{M N_{0}}} \frac{1}{\mathrm{Ac}}
$$

Note that for fixed $M$ and $N_{0}$, the error decreases as the compressibility decreases (i.e., as the sound speed increases) since the density fluctuations are smaller.

Finally we consider the measurement of temperature. First we should remark that the measurement of instantaneous temperature is subtle, even in a dilute gas. But given that temperature is measured correctly, equilibrium statistical mechanics gives the variance in the temperature fluctuations to be

$$
\left\langle\delta T^{2}\right\rangle=\frac{k T_{0}^{2}}{c_{V} N_{0}}
$$

where $c_{V}$ is the heat capacity per particle at constant volume. The fractional error in the estimate of the temperature is

$$
E_{T}=\frac{\sigma_{T}}{T_{0}}=\frac{\sqrt{\left\langle\delta T^{2}\right\rangle}}{T_{0} \sqrt{M}}=\frac{1}{\sqrt{M N_{0}}} \sqrt{\frac{k}{c_{V}}}
$$

Because the fluctuations are smaller, the error in the temperature is smaller when the heat capacity is large; note that for a monatomic dilute gas $c_{V}=\frac{3}{2} k$. 
The fractional error in the density and temperature depend only on the density and temperature of the fluid and are independent of the flow speed. Although typically undesirable in isothermal low speed flows, density and temperature gradients develop due to compressibility and viscous heating effects. The magnitude of these effects is proportional to the square of the Mach number, making them particularly challenging to resolve. Consider for example the case of viscous heat dissipation: if we express the local temperature as $T_{0}=T_{G}+\Delta T$, where $T_{G}$ is the global mean temperature, then the fractional error in the temperature deviation can be approximated by

$$
E_{\Delta T}=\frac{\sigma_{T}}{|\Delta T|} \approx E_{T}\left|\frac{T_{G}}{\Delta T}\right| \propto \frac{E_{T}}{\mathrm{Ma}^{2}}
$$

assuming $\left|\Delta T / T_{G}\right| \propto\left(\nabla u_{0}\right)^{2} \propto \mathrm{Ma}^{2}$. Although this expression is only approximate, it serves to highlight the typical scaling of signal-to-noise ratios found in low speed flows.

A final note: In DSMC simulations one considers each particle as "representing" a large number of molecules in the physical system. In all the expressions given here, $N_{0}$ is the number of particles used by the simulation so the fluctuations can be reduced by using larger numbers of particles (i.e., using a lower molecule-to-particle ratio).

\section{Simulations}

We performed DSMC simulations to verify the validity of the expressions derived above. Standard DSMC techniques $[2,3]$ were used to simulate flow of gaseous argon (molecular mass $m=6.63 \times 10^{-26} \mathrm{~kg}$, hard sphere diameter $\sigma=3.66 \times$ $10^{-10} \mathrm{~m}$ ) in a two-dimensional channel (length $L$ and height $H$ ). The simulation was periodic in the $x$ direction (along the channel axis). The two walls at $y=0$ and $y=H$ were fully accommodating and smooth with fixed temperature $T_{0}=$ $273 \mathrm{~K}$. The simulation was also periodic in the third (homogeneous) direction. A constant acceleration was applied to the particles to produce Poiseuille flow in the $x$ direction with maximum velocity at the centerline $u_{0}^{\max } \approx 2 \mathrm{~m} / \mathrm{s}$. The equilibrium density was $\rho_{0}=1.78 \mathrm{~kg} / \mathrm{m}^{3}$ and approximately 40 particles per cell were used. The cell size used was $\Delta x=\lambda_{0} / 3$ where $\lambda_{0}$ is the reference mean free path. The timestep was $\Delta t=\lambda_{0} /\left(7.5 v_{0}\right)$ where $v_{0}=\sqrt{2 k T_{0} / m}=337 \mathrm{~m} / \mathrm{s}$ is the most probable speed. To ensure that the samples taken were independent, one sample every 250 timesteps was taken starting after 1 million timesteps ensuring that the system was in steady state.

Figures 1-3 show the measured fractional error, obtained from the standard deviation of cell values in the $x$ and $z$ directions. The velocity profile in Poiseuille flow is parabolic so the fractional error in the velocity measurement is minimum at the centerline (see Fig. 1). The number of particles was nearly constant everywhere in the system so the fractional errors in density and temperature are nearly constant. In all cases, the simulation measurements are in good agreement with the theoretical predictions. 


\section{Conclusions}

We have presented expressions for the statistical error in estimating the velocity, density and temperature in molecular simulations. These expressions were validated for flow of a dilute gas in a two-dimensional channel using the direct simulation Monte Carlo technique. Despite the non-equilibrium nature of the validation experiment, good agreement is found between theory and simulation, verifying that modifications to non-equilibrium results are very small. We thus expect these results to hold for general non-equilibrium applications of interest. The validity of the above expressions for dense fluid flows is currently under investigation.

\section{Acknowledgements}

The authors wish to thank M. Malek-Mansour for helpful discussions. The authors would also like to thank Xabier Garaizar for making this work possible through the computer resources made available to the them, and Dr. Kyran Mish, Director, Center for Computational Engineering, Lawrence Livermore National Laboratory, for financial support (US Department of Energy, W-7405ENG-48). This work was also supported in part by a grant from the European Commission DG12 (PSS*1045).

\section{References}

[1] Allen MP, Tildesley DJ. Computer Simulation of Liquids. Oxford: Clarendon Press, 1987.

[2] Bird GA., 1994. Molecular Gas Dynamics and the Direct Simulation of Gas Flows. Oxford: Clarendon Press, 1994.

[3] Alexander FJ, Garcia AL. The Direct Simulation Monte Carlo Method. Computers in Physics 1997; 11, 588-593.

[4] Malek-Mansour M, Garcia AL, Lie GC, Clementi E. Fluctuating Hydrodynamics in a Dilute Gas. Physical Review Letters 1987;58 874-877; Garcia AL, Malek-Mansour M, Lie G, Mareschal M, Clementi E, Hydrodynamic fluctuations in a dilute gas under shear. Physical Review A 1987;36, 43484355 .

[5] Landau LD, Lifshitz EM. Statistical Mechanics. Oxford: Pergamon Press, 1980. 


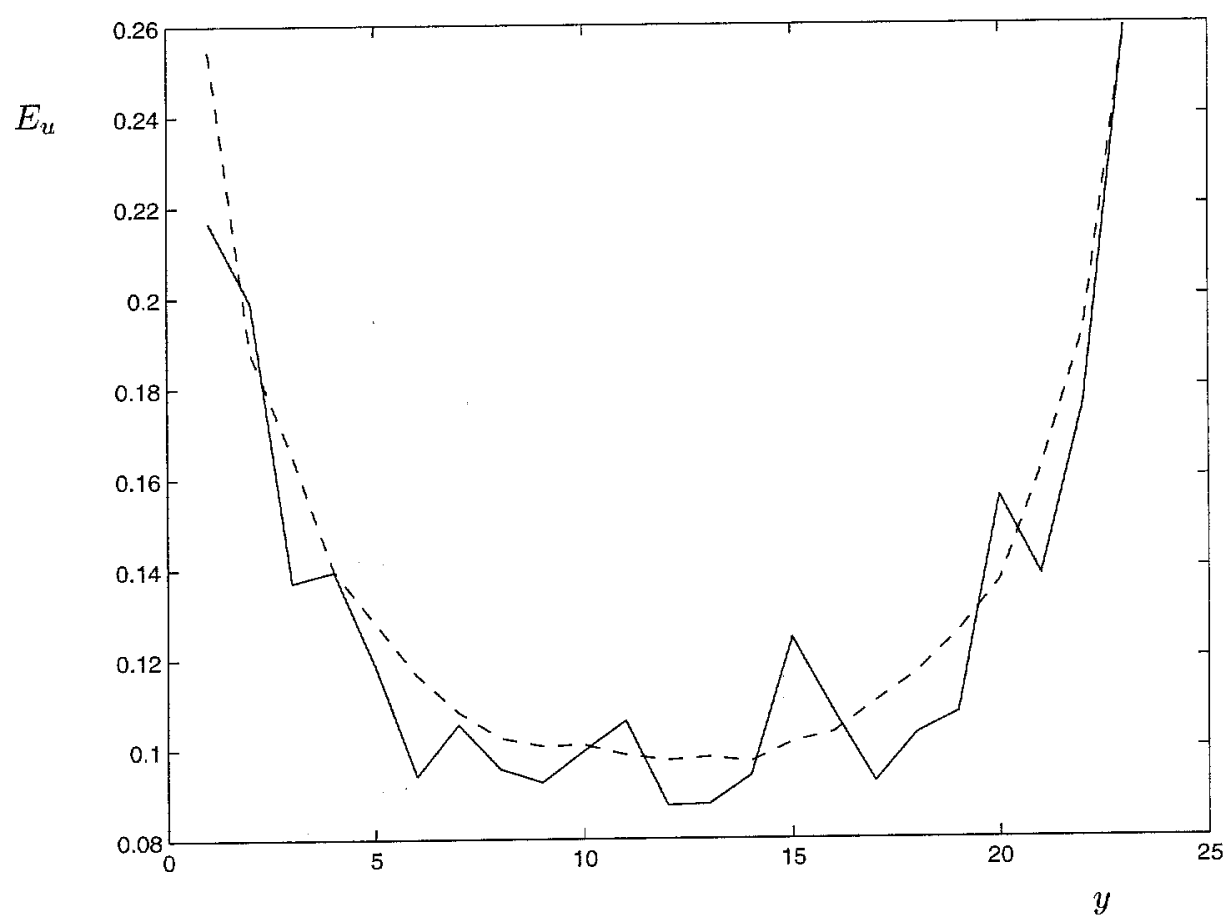

Figure 1: Fractional error in velocity for Poiseuille flow in a channel as a function of the transverse channel coordinate, $y$. The dashed line denotes equation (3) and the solid line denotes DSMC simulation results. 


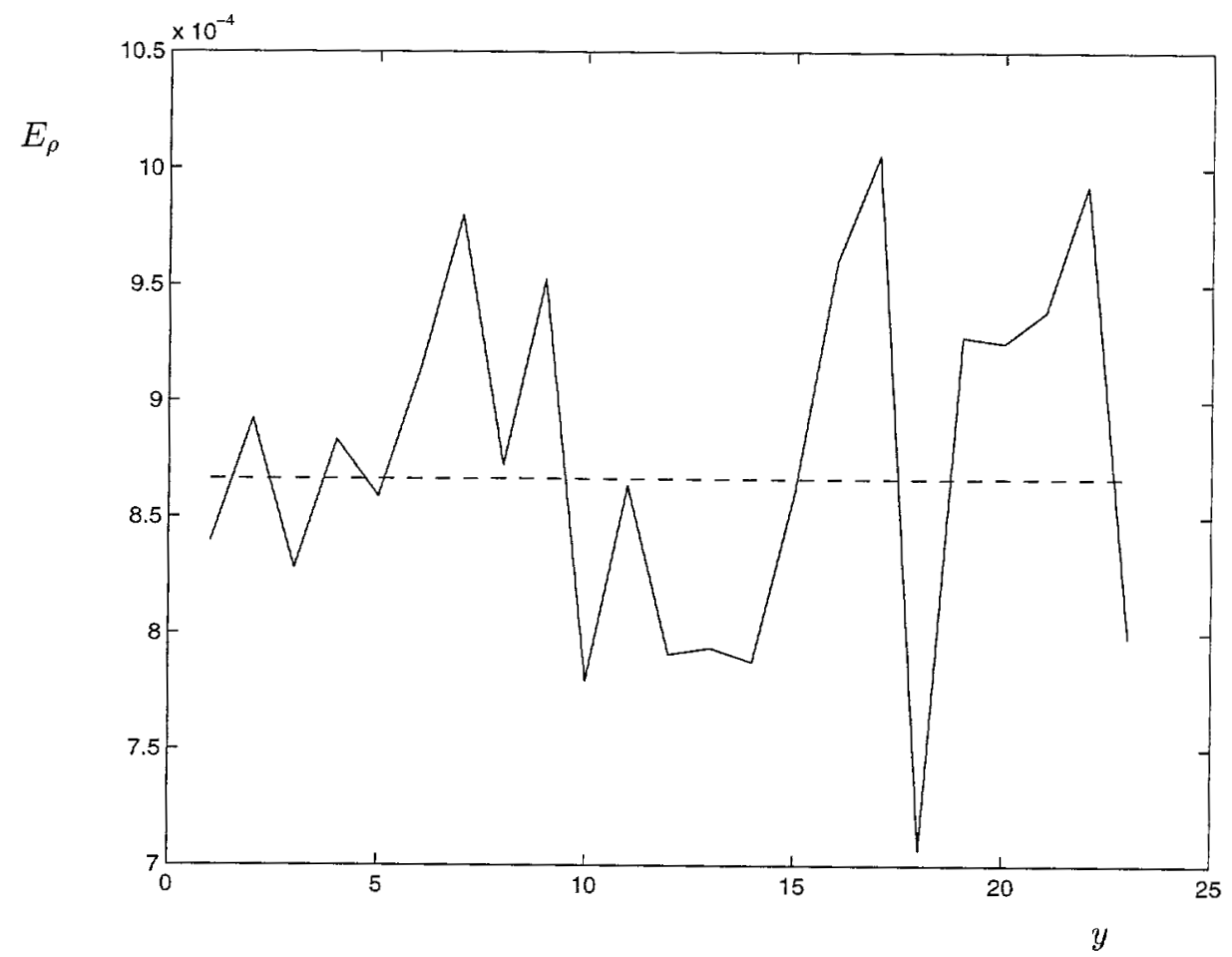

Figure 2: Fractional error in density for Poiseuille flow in a channel as a function of the transverse channel coordinate, $y$. The dashed line denotes equation (8) and the solid line indicates DSMC simulation results. 


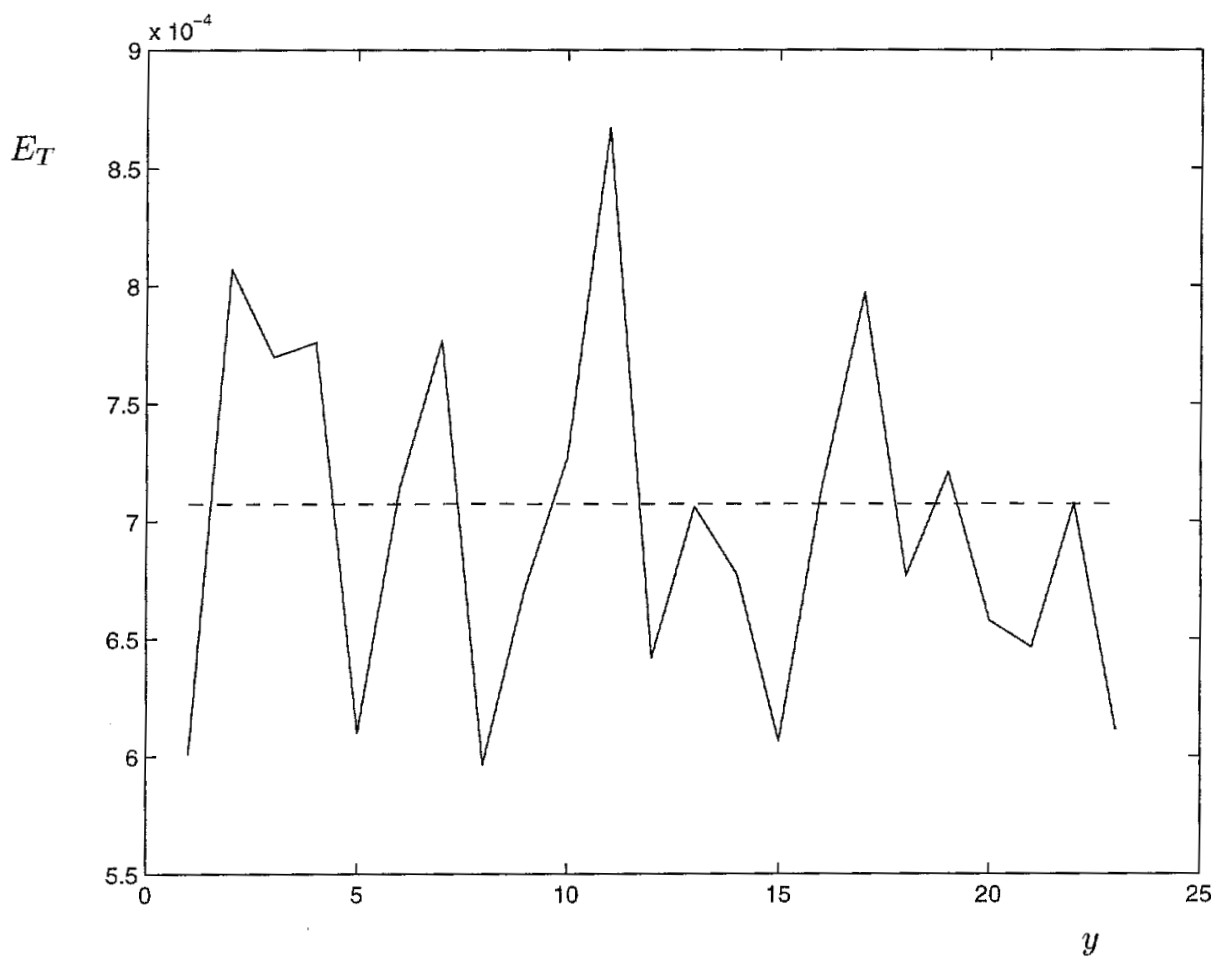

Figure 3: Fractional error in temperature for Poiseuille flow in a channel as a function of the transverse channel coordinate, $y$. The dashed line denotes equation (10) and the solid line indicates DSMC simulation results. 